\title{
Research cardiac magnetic resonance imaging in end stage renal disease - incidence, significance and implications of unexpected incidental findings
}

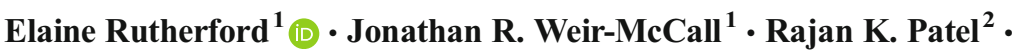 \\ J. Graeme Houston ${ }^{1}$ - Giles Roditi ${ }^{3}$ • Allan D. Struthers ${ }^{1}$ • Alan G. Jardine ${ }^{2}$ • \\ Patrick B. Mark ${ }^{2}$
}

Received: 6 October 2015 /Revised: 4 January 2016 / Accepted: 8 February 2016 /Published online: 7 April 2016

(C) The Author(s) 2016. This article is published with open access at Springerlink.com

\begin{abstract}
Objectives Left ventricular mass (LVM) at cardiac magnetic resonance imaging (CMR) is a frequent end point in clinical trials in nephrology. Trial participants with end stage renal disease (ESRD) may have a greater frequency of incidental findings (IF). We retrospectively investigated prevalence of IF in previous research CMR and reviewed their subsequent impact on participants.

Methods Between 2002 and 2006, 161 ESRD patients underwent CMR in a transplant assessment study. Images were used to assess LV mass and function. In the current study a radiologist reviewed the scans for IF. Review of patient records determined the subsequent clinical significance of IF. Results There were 150 IF in 95 study participants. Eightyfour $(56 \%)$ were new diagnoses. One hundred and two were non-cardiac. Fifteen were suspicious of malignancy. There was a clinically significant IF for $14.9 \%$ of the participants. In six cases earlier identification of an IF may have improved quality of life or survival.

Conclusions Without radiology support clinically important IF may be missed on CMR. Patients undergoing CMR in trials should be counselled about the frequency and implications of
\end{abstract}

Elaine Rutherford

e.rutherford@dundee.ac.uk

1 Division of Cardiovascular and Diabetes Medicine, Ninewells Hospital, Dundee DD1 9SY, UK

2 Institute of Cardiovascular and Medical Sciences, British Heart Foundation Building, Glasgow Clinical Research Centre, Glasgow G12 8TA, UK

3 Department of Radiology, Glasgow Royal Infirmary, NHS Greater Glasgow and Clyde, 84 Castle Street, Glasgow G4 0ET, UK
IF. Patients with ESRD have a higher prevalence of IF than reported in other populations. Nephrology studies require mechanisms for radiologist reporting and strategies for dealing with IF.

Key Points

- Incidental findings on research cardiac magnetic resonance imaging can have significant consequences.

- We considered incidental findings in historical renal cardiac resonance imaging clinical trials.

- Incidental findings are common and important in the chronic kidney disease population.

- Without radiology support, clinically significant incidental findings may be missed on imaging.

- Study protocols, approvals and consent processes should take account of possible findings.

Keywords Chronic kidney disease · Clinical trials · MRI · Incidental findings $\cdot \mathrm{CMR}$

$\begin{array}{ll}\text { Abbreviations } \\ \text { CKD } & \text { Chronic kidney disease } \\ \text { CMR } & \text { Cardiac magnetic resonance imaging } \\ \text { ESRD } & \text { End stage renal disease } \\ \text { HOCM } & \text { Hypertrophic obstructive cardiomyopathy } \\ \text { IF } & \text { Incidental findings } \\ \text { LVH } & \text { Left ventricular hypertrophy } \\ \text { LVM } & \text { Left ventricular mass }\end{array}$

\section{Introduction}

Reduction in cardiac morbidity and mortality is of great importance in nephrology. Within the chronic kidney disease 
(CKD) population, increased left ventricular mass (LVM) is well established as a surrogate marker of cardiovascular risk [1]; therefore, reduction in LVM assessed by cardiac magnetic resonance imaging (CMR) is a frequent primary end point in nephrology clinical trials [2-9].

Patients consenting to participate in clinical trials undergo CMR that would not otherwise be performed. Standard CMR imaging techniques commonly capture windows out with the area of the heart including the abdomen and thorax. Clearly incidental findings (IF) on images obtained for research may have unexpected clinical consequences. The prevalence of unexpected findings in clinically indicated CMR has been well documented [10-13]. While the reported incidence of incidental non-cardiac findings in non-renal populations varies from around 7-27 \%, the rate of clinically significant IF is much lower than this at around 1-7 \% [10-13]. The rate of IF in CMR performed for research purposes is less well established [14]. However, findings in research CMR are important and current radiology guidelines reflect this [15].

Trial participants with CKD may be at increased risk of having clinically significant IF. In those with end stage renal disease (ESRD) the risk may be even greater as these individuals are likely to have a larger burden of ill health. This potential increased risk of IF could have ethical, clinical and financial implications for future CMR nephrology studies and their participants. The prevalence of IF in CMR in patients with ESRD requiring dialysis is unknown. To investigate this, we retrospectively determined the prevalence of IF in previous research CMR and reviewed its potential subsequent impact on patient care.

\section{Subjects and methods}

\section{Participants}

Between 2002 and 2006, 161 patients underwent CMR as part of a prospective single centre research study considering cardiac risk and transplantation assessment under the care of the Renal Transplant Unit at the Western Infirmary Glasgow [8, 9, $16,17]$. All participants had CKD stage 5 (estimated glomerular filtration rate $<15 / \mathrm{min}$ ) and were receiving peritoneal or haemodialysis, or were within 6 months of requiring it (predialysis). Participants had no contraindications to CMR but were otherwise an unselected group. The study was approved by the local ethics committee. Prior to imaging, all participants provided written informed consent.

\section{CMR acquisition and analysis}

CMR imaging was performed using a 1.5-Tesla magnetic resonance imaging scanner (Sonata, Siemens Erlangen, Germany). In the original study, images were used by our research group solely to assess cardiac parameters, in particular LVM and cardiac function. For those on haemodialysis, the scans were consistently performed 24 hours after the end of the last dialysis session. Following acquisition of localizer images using a gradient echo sequence (FLASH: $\mathrm{TR}=3.25 \mathrm{~ms} ; \mathrm{TE}=1.6 \mathrm{~ms} ; \mathrm{FA}=25^{\circ}$; pixel area $=1.4 \mathrm{~mm}$ by $1.4 \mathrm{~mm}$; slice thickness $=8 \mathrm{~mm}$; FOV $=360 \mathrm{~mm}$ ) a steadystate free precession (TrueFISP) sequence was used to acquire cine images in three long axis planes (vertical long axis, horizontal long axis, left ventricular outflow tract) followed by short axis sequences from the atrioventricular ring to the apex. Cine sequences were obtained with the following acquisition parameters: $\mathrm{TR}=3.14 \mathrm{~ms} ; \mathrm{TE}=1.6 \mathrm{~ms} ; \mathrm{FA}=60^{\circ}$; pixel area $=2.2 \mathrm{~mm}$ by $1.3 \mathrm{~mm}$; slice thickness $=8 \mathrm{~mm}$; $\mathrm{FOV}=340 \mathrm{~mm}$. Evidence of prior infarcts was sought on short axis late gadolinium enhancement sequences. All these studies were performed prior to any link between the use of gadolinium contrast agents with nephrogenic systemic fibrosis (NSF) was established in CKD patients [18, 19]. Late gadolinium enhancement imaging was acquired using a breathhold segmented turbo fast low angle shot (FLASH) inversion-recovery sequence with the following acquisition parameters: $\mathrm{TR}=11.6 \mathrm{~ms} ; \mathrm{TE}=4.3 \mathrm{~ms} ; \mathrm{FA}=20^{\circ}$; pixel area $=2.2 \mathrm{~mm}$ by $1.3 \mathrm{~mm}$; slice thickness $=8 \mathrm{~mm}$; $\mathrm{FOV}=340 \mathrm{~mm}$. From March 2004, in order to assess aortic indices, a transverse HASTE stack of the thorax was adopted into our imaging protocol $(\mathrm{TR}=600 \mathrm{~ms}$; $\mathrm{TE}=24 \mathrm{~ms}$; $\mathrm{FA}=160^{\circ}$; pixel area $=1.4 \mathrm{~mm}$ by $1.4 \mathrm{~mm}$; slice thickness $=8 \mathrm{~mm}$; FOV $=360 \mathrm{~mm}$ ). From this a 'candy cane' TrueFISP sequence of the thoracic aorta was obtained. An axial oblique through plane phase contrast sequence was also obtained through the thoracic aorta at the level of the right pulmonary artery. This was performed in 113 patients. In the current study, an experienced radiologist from another centre reviewed all images for IF. The reviewing radiologist was blinded to patient identity, outcomes, original scans and subsequent imaging.

\section{IF classification}

Findings were categorized as cardiac and non-cardiac IF. Cardiac findings were sub-classified as valve, pericardial or findings suggestive of cardiomyopathy. Multiple valve abnormalities on a scan were considered a single finding. Abnormalities in ventricular mass, size, perfusion or function were not considered IF unless consistent with a previously undiagnosed non-uraemic cardiomyopathy.

Non-cardiac findings were subcategorized as lung/ mediastinal, pleural effusions, gastrointestinal, hepatic/renal cysts and 'other' findings. Renal atrophy was not considered an IF. Hepatic and renal cysts were not considered IF, if there was a history of polycystic kidney disease. 


\section{Establishing clinical significance}

Current and historical electronic clinical and radiology records for all participants with one or more IF were reviewed by a clinician who was not involved in subsequent patient care. The end of the follow-up period for establishing IF significance was considered as the date of death or the date clinical records were reviewed (if later). Whether a finding raised the possibility of malignancy was noted. Figure 1 details the process followed for determining the significance of IF. The clinical significance of IF was determined by answering three questions:

- Did the finding represent a new diagnosis?

Cases where it was not possible to determine if the finding represented a new diagnosis or not were not considered new diagnoses. Pleural effusions were only considered to be a new diagnosis if they were unilateral and at least moderately sized.

- Did, or would, the finding have altered clinical management at the time of imaging (e.g., further investigation or referral)?

IF were considered significant if they altered, or would have altered patient management when recognised.

- Would earlier knowledge of the finding have feasibly altered the patient's clinical course and potentially improved quality of life or survival?

This question was only asked of significant IF.

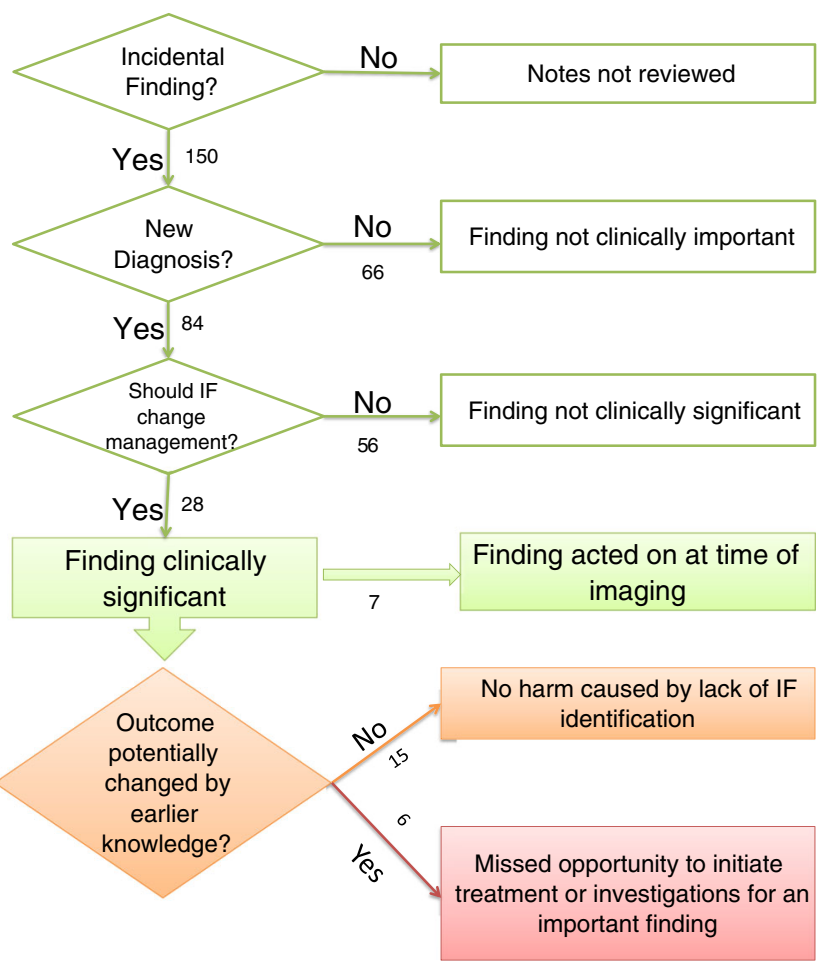

Fig. 1 Flow diagram showing the number and significance of findings at each level of importance. The numbers represent the number of findings at each point in the process

\section{Statistics}

Statistical analysis was performed using SPSSv21 (IBM, Armonk, NY, USA). For normally distributed data, a t-test was used to compare means across groups. For nonparametric data, a Mann-Whitney test was used. Regression analysis determined whether the presence of an IF was predictive of poorer survival.

\section{Results}

\section{Participant characteristics}

Mean age at time of imaging was 52.3 years (range 25-77 years). A full breakdown of baseline patient demographics and available clinical data is available in Table 1. As in our original research, there was a high prevalence of traditional cardiovascular risk factors [9]. At the end of follow-up, $50.3 \%$ of participants were alive. Median follow-up duration was 10.0 years (range 1 day-12.4 years).

Table 1 Baseline demographic and clinical data for participants

\begin{tabular}{lc}
\hline Variable & Total $\left(161\right.$ participants $\left.^{\mathrm{a}}\right)$ \\
\hline Age at CMR (years) & 52.3 \\
Male & $104(64.6 \%)$ \\
Body surface area $\left(\mathrm{m}^{2}\right)$ & $1.84( \pm 0.23)$ \\
BMI $\left(\mathrm{kg} / \mathrm{m}^{2}\right)$ & $26.02( \pm 5.07)$ \\
Systolic blood pressure $(\mathrm{mmHg})$ & $138( \pm 25)$ \\
Diastolic blood pressure $(\mathrm{mmHg})$ & $82( \pm 13)$ \\
Renal replacement therapy & \\
Haemodialysis $(\%)$ & 44.7 \\
Peritoneal dialysis $(\%)$ & 35.4 \\
Pre-dialysis (\%) & 19.9 \\
Diabetes mellitus $(\%)$ & 31 \\
Hypertension $(\%)$ & 50.6 \\
Previous myocardial infarction $(\%)$ & 5.7 \\
Ischaemic heart disease $(\%)$ & 8.2 \\
Cerebrovascular disease $(\%)$ & 4.4 \\
Peripheral vascular disease $(\%)$ & 2.5 \\
Dyslipidaemia $(\%)$ & 24.7 \\
Never Smoker $(\%)$ & 53.2 \\
Current/ Ex smoker $(\%)$ & 46.8 \\
Haemoglobin $(\mathrm{g} / \mathrm{dL})$ & $11.45( \pm 1.97)$ \\
Corrected calcium $(\mathrm{mmol} / \mathrm{l})$ & $2.29( \pm 0.31)$ \\
Albumin $(\mathrm{g} / \mathrm{dl})$ & $39.2( \pm 4.8)$ \\
Phosphate (mmol/l) & $1.68( \pm 0.5)$ \\
CMR ejection fraction $(\%)$ & $67.5( \pm 11.2)$ \\
CMR left ventricular mass index $\left(\mathrm{g} / \mathrm{m}^{2}\right)$ & $91.4( \pm 27.6)$ \\
\hline & \\
\hline
\end{tabular}

${ }^{a}$ Full clinical demographic data was not available for 5 participants 
Participants with an IF tended to be older than those without, although this was not statistically significant (mean 53.6 years vs 50.4 years, respectively, $P=0.08$ ). There was no difference in prevalence of IF across modality of renal replacement therapy or any other baseline characteristic.

\section{Number and significance of findings}

Review of the $161 \mathrm{CMR}$ scans revealed 150 IF in 95 patients (59\% of patients). Eighty-four (56\%) of the IF in 66 patients would have resulted in a new diagnosis.

There were 28 significant IF that warranted further investigation or a change in patient management. Twenty-four participants had one or more significant findings (14.9\% participants). Seven significant IF were identified and acted on at the time of imaging. There were 21 cases of potentially significant IF where the finding was not identified at the time of imaging. In 15 of these cases, record review confirmed that lack of intervention ultimately did not harm patient outcome. In six cases, affecting four $(2.5 \%)$ participants, lack of recognition of the finding was a missed opportunity to initiate investigations or treatment for a finding that later became clinically significant. Earlier identification of these findings may have improved the patients' quality of life or survival (Table 2). Figure 1 illustrates the process of determining the significance of the IF and the number of findings at each level of significance.

\section{Influence of imaging protocol on IF rate}

There was no difference in the rate of IF in those who had a HASTE stack performed as part of their imaging sequence and those who did not. Contrast administration was important for the late gadolinium enhancement IF, where it contributed to the diagnosis of Hypertrophic Obstructive Cardiomyopathy (HOCM). In an additional case, perfusion sequences were useful to characterize a hepatic lesion, which was indeterminate on other sequences, but showed classical enhancement of a haemangioma. Other than these scenarios, contrast added little to the diagnostic yield.

\section{Cardiac findings}

There were 48 cardiac IF, 34 (70.8\%) were new diagnoses. Of these, nine $(26.5 \%)$ did, or would have warranted a change in patient management when recognized. On one occasion earlier identification of a finding of HOCM may have led to an earlier change in the patient's treatment and potentially improved quality of life.

\section{Valve findings}

Valve disease was the most common cardiac IF (34 cases, 70.8 $\%$ of cardiac findings). Table 3 gives a breakdown of which valves were affected. Eleven findings $(32.4 \%)$ of valve disease were known about prior to CMR imaging. Valve disease was a new diagnosis in 23 cases, in 15 (65.2\%) of these cases, there was clear documentation that the abnormality was identified at the time of scanning. In nine of these 15 cases, no changes to subsequent patient management were necessary. In the remaining six cases, the diagnosis had a direct impact on patient care (e.g., referral for valve replacement).

There were eight cases where there was no specific mention of valve disease on available documentation. In seven of these cases, it was clear from note review that these lesions would not have significantly altered subsequent patient management. In a single case, knowledge of the valve disease - severe aortic stenosis - would have changed management, but there was in practice insufficient time between scanning and the patient's death (less than 48 hours) for any changes to be effected.

\section{Cardiomyopathy}

The presence and characteristic findings of uraemic cardiomyopathy in this population group have been well described with

Table 2 Summary of incidental findings where earlier detection may have improved quality of life or survival

\begin{tabular}{|c|c|c|}
\hline Finding on CMR & CMR year & Comment \\
\hline Hypertrophic obstructive cardiomyopathy & 2004 & Eventually diagnosed 2011 following progression of symptoms \\
\hline Lung lesion highly suspicious of malignancy ${ }^{\text {a }}$ (Fig. 5) & 2004 & Died of possible malignancy 2008 \\
\hline $\begin{array}{l}\text { Oesophageal lesion highly suspicious of malignancy }{ }^{\mathrm{a}} \\
\text { (Fig. 5) }\end{array}$ & 2004 & Died of possible malignancy 2008 \\
\hline Large unilateral pleural effusion & 2004 & Lower lobe lobectomy for presumed adenocarcinoma 2007 \\
\hline Multiple suspicious splenic lesions ${ }^{\mathrm{b}}$ (Fig. 4) & 2004 & $\begin{array}{l}\text { Picked up incidentally on abdominal CT } 2013 \text { - resulted in } 6 \text { month unnecessary } \\
\text { suspension from transplant list }\end{array}$ \\
\hline Multiple suspicious liver lesions ${ }^{\mathrm{b}}$ & 2004 & $\begin{array}{l}\text { Picked up incidentally on abdominal CT } 2013 \text { - resulted in } 6 \text { month unnecessary } \\
\text { suspension from transplant list }\end{array}$ \\
\hline
\end{tabular}

\footnotetext{
${ }^{a}$ lesions were in same participant. Cause of death on death certificate was 'cardiac arrest' but participant's clinical team felt malignancy may have been the actual cause of death - post mortem was not pursued

${ }^{\mathrm{b}}$ lesions were in same participant
} 
Table 3 Breakdown of type of incidental valve lesions

\begin{tabular}{lc}
\hline Valve lesion & $\begin{array}{l}\text { Number of participants } \\
\text { with findings }\end{array}$ \\
\hline Aortic regurgitation & 9 \\
Aortic stenosis & 22 \\
Mitral regurgitation & 10 \\
Tricuspid regurgitation & 5 \\
\hline
\end{tabular}

${ }^{\text {a }} 12$ participants had more than one valve lesion

Left Ventricular Hypertrophy (LVH) and dilatation being prominent findings $[9,20]$. The reviewing radiologist considered that ten participants had changes that could be broadly consistent with a cardiomyopathy. Changes consistent with cardiomyopathy accounted for $6.7 \%$ of all IF and affected $6.2 \%$ of the study population. In four cases this was already known about prior to CMR. In six cases this represented a new diagnosis, in two of these new cases, potential cardiomyopathy identification changed immediate patient management. In one case, the findings were noted but no changes to patient management were appropriate. In three cases, no comment was found in historical records with regard to cardiomyopathy. Patient outcome could conceivably have been influenced in only one of these cases. In this case, the radiologist considered a 2004 scan to be consistent with HOCM - our research group interpreted these changes as showing evidence of prior ischaemia and LVH. In 2011 this patient was diagnosed with HOCM on repeat CMR following progressive symptoms consistent with the diagnosis. Although HOCM is a progressive condition, better symptom control could arguably have been achieved through earlier recognition of this IF. However these types of finding are clearly subject to interpretation in the context of uraemic cardiomyopathy, which has many features that may overlap with 'early' HOCM.

\section{Non-cardiac findings}

There were 102 non-cardiac IF affecting $43.5 \%$ of participants. Fifty non-cardiac findings $(49.0 \%)$ were classed as new diagnoses. Of these 50, $19(38.0 \%)$ warranted further investigation or a change in patient management when identified. On five occasions, lack of recognition of such a finding was a missed opportunity to initiate further investigations or treatment for a finding that later became clinically significant (Table 2). Earlier identification of these findings may have led to a change in the patient's clinical course and potentially improved quality of life or survival. Table 4 gives a breakdown of the different categories of non-cardiac findings and their ultimate impact on patient care.

\section{Findings suggestive of malignancy}

All of the 14 non-cardiac IF that would have triggered further investigation, but ultimately did not alter the participant's clinical course, were suspicious of malignancy. In total there were 15 IF that were suspicious of malignancy. These IF and patient outcomes are detailed in Table 5.

\section{Influence of IF on length of survival}

The presence or absence of an IF on imaging did not have any statistically significant impact on length of survival. See Fig. 2 - Kaplan Meier Death-censored survival. Estimated mean survival: No IF 8.4 (95 \% CI 7.5-9.3) years vs with an IF 8.3 (95\% CI 7.4-9.2) years.

\section{Discussion}

This is the first study looking at the rate of IF on CMR performed for research purposes in the ESRD population. Our study highlights that IF in research CMR in this population are
Table 4 Breakdown of the number of different types of non-cardiac findings and their impact on patient care

\begin{tabular}{lccll}
\hline Finding classification & $\begin{array}{l}\text { Total number } \\
\text { of findings }\end{array}$ & New diagnosis & $\begin{array}{l}\text { Would have changed } \\
\text { management if identified } \\
\text { but did not alter clinical } \\
\text { course }\end{array}$ & $\begin{array}{l}\text { Identification } \\
\text { could feasibly } \\
\text { have altered } \\
\text { clinical course }\end{array}$ \\
\hline Lung or mediastinal finding & 11 & 9 & 6 & 1 \\
Pleural effusion & 15 & 4 & 3 & 1 \\
GI tract & 19 & 12 & 1 & 3 \\
Hepatic/Renal cyst & 50 & 18 & 1 & 0 \\
$\quad$ Not known PKD) & 7 & 7 & 3 & 0 \\
Other significant finding & 102 & 50 & 14 & 5 \\
Total & & & & \\
\hline
\end{tabular}


Table 5 Summary of incidental findings potentially suspicious of malignancy

\begin{tabular}{|c|c|c|c|c|}
\hline Finding & Description of Finding & $\begin{array}{l}\text { CMR } \\
\text { Year }\end{array}$ & $\begin{array}{l}\text { Comment on outcome of } \\
\text { finding }\end{array}$ & $\begin{array}{l}\text { Year of } \\
\text { Death/ } \\
\text { Last } \\
\text { follow up }\end{array}$ \\
\hline 1 & $\begin{array}{l}3 \text { nodules in left lung with } \\
\text { associated } \\
\text { adenopathy }^{\mathrm{a}}\end{array}$ & 2004 & $\begin{array}{l}\text { Never identified, participant } \\
\text { may have died of malignancy }\end{array}$ & 2008 \\
\hline 2 & $\begin{array}{l}33 \mathrm{~mm} \text { mass in region of } \\
\text { oesophagus }^{\mathrm{a}}\end{array}$ & 2004 & $\begin{array}{l}\text { Never identified, participant } \\
\text { may have died of malignancy }\end{array}$ & 2008 \\
\hline 3 & Multiple splenic lesions $^{b}$ & 2004 & $\begin{array}{l}\text { Identified 2013, suspended } \\
\text { from transplant list for } 6 \\
\text { months whilst undergoing } \\
\text { serial imaging }\end{array}$ & $\begin{array}{l}\text { Alive } \\
2015\end{array}$ \\
\hline 4 & $\begin{array}{l}\text { Multiple liver cysts, one of atypical } \\
\text { signal requiring further } \\
\text { investigation }^{b}\end{array}$ & 2004 & $\begin{array}{l}\text { Identified 2013, suspended } \\
\text { from transplant list for } 6 \\
\text { months whilst undergoing } \\
\text { serial imaging }\end{array}$ & $\begin{array}{l}\text { Alive } \\
2015\end{array}$ \\
\hline 5 & Multiple splenic lesions & 2004 & $\begin{array}{l}\text { Never identified, was awaiting } \\
\text { outpatient surgical review for } \\
\text { abdominal pain but died day } \\
\text { after scan }\end{array}$ & 2004 \\
\hline 6 & $\begin{array}{l}\text { Mediastinal and Hilar } \\
\text { Lymphadenopathy }\end{array}$ & 2005 & $\begin{array}{l}\text { Never identified, no further } \\
\text { imaging }\end{array}$ & 2007 \\
\hline 7 & $14 \mathrm{~mm}$ Adrenal Mass & 2004 & $\begin{array}{l}\text { Never identified, had } \\
\text { abdominal CT } 2005 \text { when } \\
\text { lesion was not seen }\end{array}$ & 2005 \\
\hline 8 & $\begin{array}{l}\text { Enlarged Superior Mediastinal } \\
\text { Nodes }\end{array}$ & 2004 & $\begin{array}{l}\text { No further imaging, renal } \\
\text { transplant } 2008\end{array}$ & $\begin{array}{l}\text { Alive } \\
2015\end{array}$ \\
\hline 9 & $10 \mathrm{~mm}$ right lower lobe lung nodule & 2004 & $\begin{array}{l}\text { Never identified, no further } \\
\text { imaging since }\end{array}$ & $\begin{array}{l}\text { Alive } \\
2015\end{array}$ \\
\hline 10 & $6 \mathrm{~mm}$ right lower lobe lung nodule & 2004 & $\begin{array}{l}\text { Admitted with lobe collapse } \\
2005 \text {, was very frail with } \\
\text { multiple co-morbidities which } \\
\text { were likely to supersede this } \\
\text { finding }\end{array}$ & 2005 \\
\hline 11 & $30 \mathrm{~mm}$ minimally enhancing mass in & 2005 & Never identified, no further & Alive \\
\hline
\end{tabular}


Table 5 (continued)

\begin{tabular}{|c|c|c|c|c|}
\hline & left axilla & & imaging. Transplant 2007 & 2015 \\
\hline 12 & $\begin{array}{l}6 \mathrm{~mm} \text { opacification/nodule in right } \\
\text { upper lobe of lung }\end{array}$ & 2005 & $\begin{array}{l}\text { Never identified, multiple } \\
\text { further CXRs since, transplant } \\
2012\end{array}$ & $\begin{array}{l}\text { Alive } \\
2015\end{array}$ \\
\hline 13 & Right adrenal mass & 2005 & $\begin{array}{l}\text { Incidentally discovered on } \\
\text { abdominal CT 2008, } \\
\text { subsequent serial imaging } \\
\text { confirmed adenoma }\end{array}$ & 2010 \\
\hline 14 & Large unilateral pleural effusion & 2004 & $\begin{array}{l}\text { Not present on subsequent } \\
\text { imaging }\end{array}$ & $\begin{array}{l}\text { Alive } \\
2015\end{array}$ \\
\hline 15 & $\begin{array}{l}\text { Multiple paraspinal soft tissue } \\
\text { masses }- \text { could represent } \\
\text { lymphoma, haematopesis or } \\
\text { neurofibromas }\end{array}$ & 2004 & $\begin{array}{l}\text { Patient had Thalassaemia } \\
\text { major with multiple blood } \\
\text { transfusions - haematopesis } \\
\text { likeliest cause. Transplant } \\
2006\end{array}$ & 2012 \\
\hline
\end{tabular}

${ }^{\mathrm{a}}$ Findings 1and 2 were in same participant, ${ }^{\mathrm{b}}$ findings 3 and 4 were in same participant

${ }^{\mathrm{c}}$ Cause of death on death certificate was 'cardiac arrest' but participant's clinical team felt malignancy may have been the actual cause of death - post mortem was not pursued

both common and have potentially serious clinical implications (Fig. 3). The prevalence of IF in our study population is greater than that in several studies looking at the rate in clinically indicated CMR $[10,11,13]$. In fact, it is more than seven times greater than the rate reported by Chan in a clinically robust study of 1534 consecutive clinically indicated CMR studies (59.0\% vs $7.6 \%$ ) [10]. This is striking as Chan's group was examining clinically indicated CMR images and one might expect the prevalence of IF in clinically indicated scans to be considerably higher than that of nonclinically indicated research scans. As our scans were undertaken purely for research purposes, we also included a limited number of cardiac IF in our initial analysis. However, even if cardiac IF are excluded, the prevalence of non-cardiac IF in our study population $(43.5 \%)$ is higher than a number of previously reported rates (range $7.6 \%-27 \%$ ) [10-13, 21].

Only one major study has a rate of IF significantly higher than ours. This study was performed specifically to determine the rate of IF on CMR in 132 volunteers revealed IF in $81 \%$ of participants [14]. However, this remarkably high prevalence rate is not truly comparable to ours as this study classed a much broader range of findings as IF (for example sternotomy wires). Their participants were also considerably older (mean age 74.2). Furthermore their study did not consider the subsequent real life clinical significance of their findings.
Not only was our rate of finding higher than in comparable studies, our study revealed a large number of clinically significant findings. Review of our images resulted in 84 new diagnoses in 161 studies. However, we have not considered all new diagnoses to be clinically significant. Whilst arguably useful to know about, asymptomatic diverticulosis or gallstones is unlikely to have any significant impact on any individual (Fig. 4).

Of much more importance to trial participants is if any IF would lead to further investigation, or to a change in their management. Like Sohns [13], we chose to classify findings as significant or not on this basis. Using this more stringent definition of a significant finding, $14.9 \%$ of our participants had a significant new IF. This is higher than in some other clinical studies where comparable definitions have been used (range 0.4-7 \%) [10-13]. Given such a high prevalence rate, it is our recommendation that all potential participants in renal studies involving CMR are counselled about the possibility of significant findings and the impact that they could have on their care prior to obtaining consent.

Uniquely in this study, follow-up of all patients with IF through review of electronic records allowed us to determine the subsequent true impact of these significant findings on patient care. In seven cases identification of a major finding at the time of imaging directly led to a significant change in 
Fig. 2 Kaplan Meier plot showing comparison of survival in those with and without any incidental findings

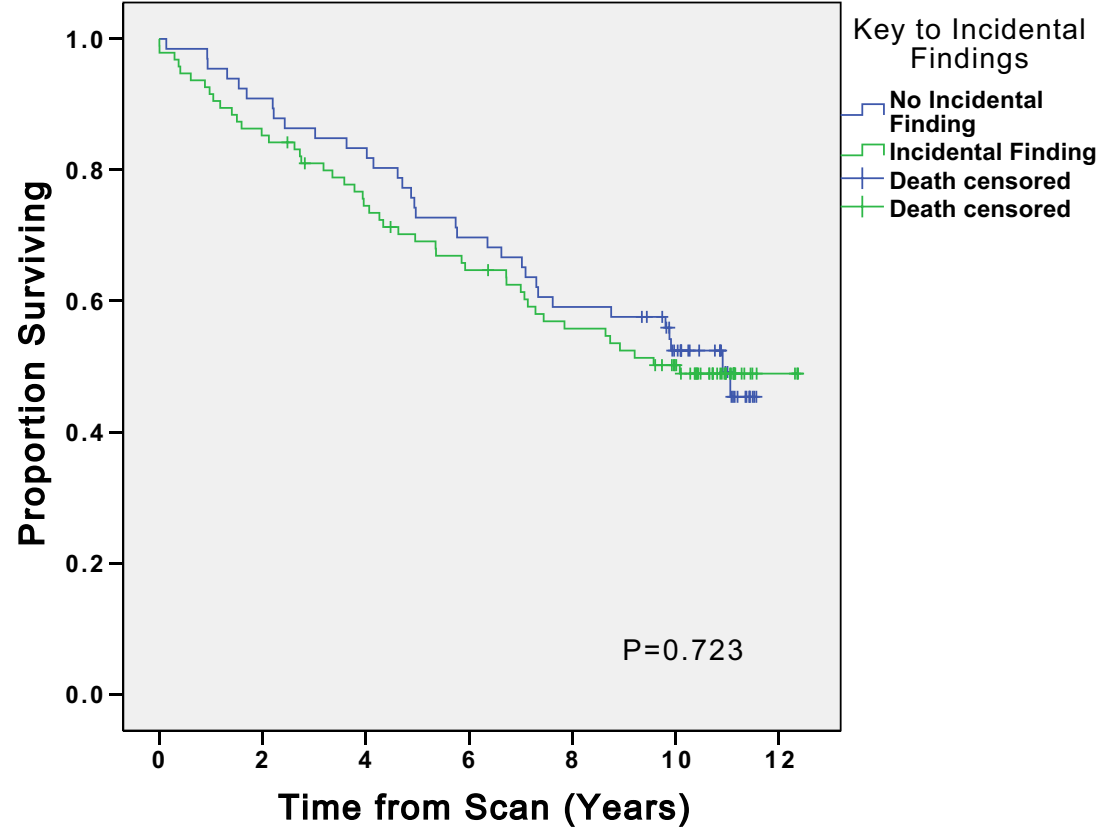

patient management (for example, aortic valve replacement). Such cases may partially explain why there was no difference in survival of those with and without findings - the actions taken as a result of a major finding at the time of imaging may have improved subsequent participant survival (Fig. 2). However, the main reason why we saw no difference in survival of the two groups is likely to be due to the confounding multiple co-morbidities of the ESRD population.

At an individual level there were cases where survival may have been influenced had the images been reported at the time of imaging. For four participants (six significant findings) the abnormality was not identified at the time of imaging and an opportunity to influence patient care at an earlier stage was missed (Fig. 5). Those six findings are detailed in Table 1. In

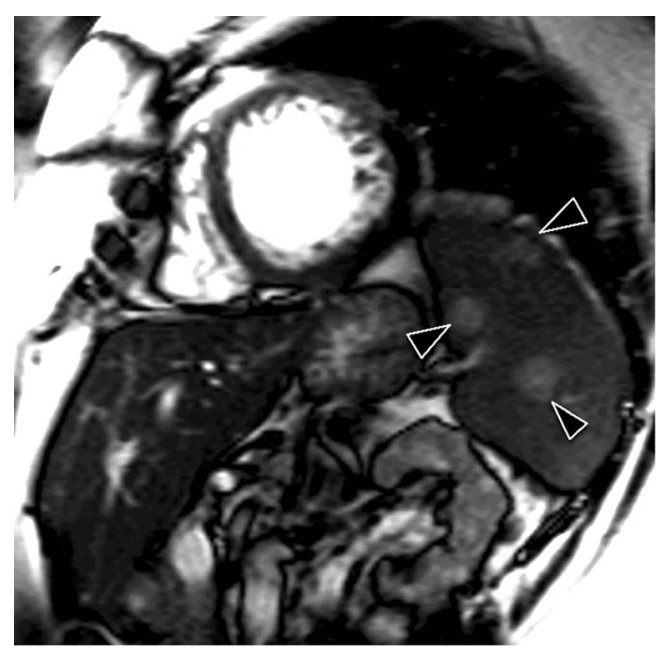

Fig. 3 Multiple high signal splenic lesions (arrow heads) are visible on this short axis cine. No prior history of malignancy was present order to ensure such opportunities are not missed in future renal studies, we recommend that all research images obtained should be reported by a suitably trained radiologist within clinically acceptable timeframes. This is in keeping with current radiology research guidelines [15].

We also wish to point out that early knowledge of IF is not always an advantage. Table 5 highlights findings that could be interpreted as being suggestive of malignancy, the findings

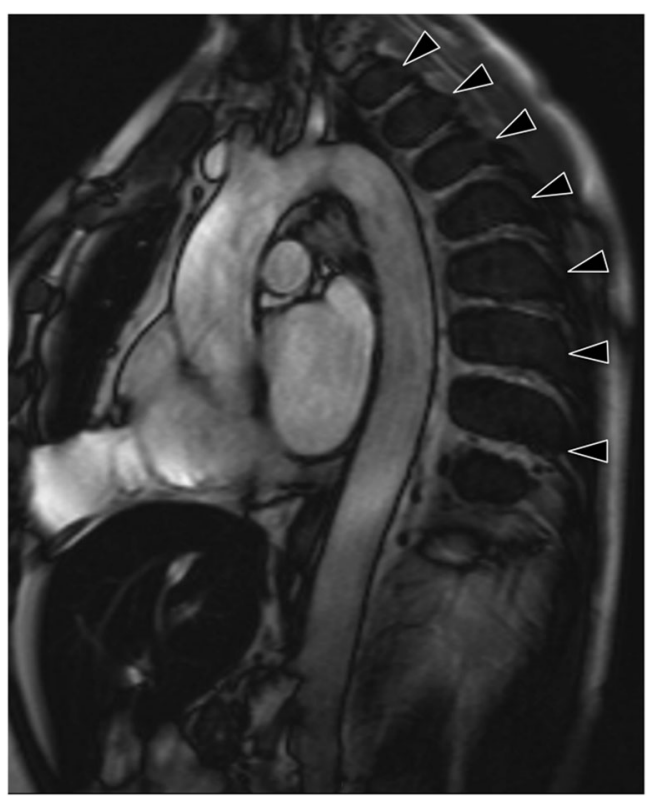

Fig. 4 Multiple regularly spaced low signal paraspinal masses (arrow heads) are evident on this candy cane view of the aorta. Note also the low signal within the liver, the combination of which is consistent with extramedullary haematopoesis in a patient with thalassaemia 
Fig. 5 Oesophageal mass/paraoesophageal adenopathy (black arrow head), upper mediastinal nodes (white arrow head) and lung nodules (arrow)

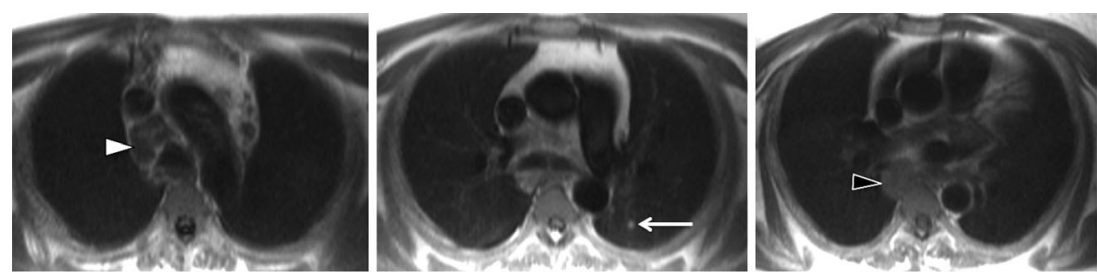

highlighted in blue were not identified at the time of imaging. Follow up of these participants revealed that the potentially suspicious finding did not impact on their clinical course. Such findings are incidentalomas and their discovery at the time of imaging may have caused considerable stress and subjected participants to unnecessary further investigations. Subjects could even have been harmed by IF identification, as a number of the findings would have resulted in arguably unnecessary transplant listing suspension until investigations were completed. This potential risk of harm should also be detailed to potential study participants prior to obtaining their consent. However, the authors of this paper believe the potential benefits of early identification of potentially significant IF far outweigh the potential risks.

Given the importance of IF within this research population, each study should have defined protocols for how IF will be managed. Ethical committees considering prospective approval of renal research studies should consider the implications of IF and ensure that there is a robust protocol for their management prior to granting study approval.

It is worth highlighting that the burden of IF in research CMR in this population is also likely to be replicated in CMR performed for clinical reasons. Current guidelines recommend that all individuals, regardless of presence or absence of symptoms, should have an echocardiogram shortly after commencing renal replacement therapy [22]. We know that increased LVM is strongly predictive of outcome in all cases, including those with ESRD [1,23]. A number of studies have shown that LVM measurement by echocardiography in this population is less robust than by CMR [24, 25]. As the cost of CMR falls and it is more widely available, its role in the clinical assessment of ESRD patients is likely to expand. Future guidelines may include assessment of LV indices with CMR. This means the frequency of incidental findings in this population will become more relevant as CMR is increasingly used for baseline clinical cardiac investigations.

Finally, the link between gadolinium based contrast used for MRI imaging and development of NSF in patients with advanced CKD, means that this study cannot be reproduced $[19,26]$. Whilst we did not find gadolinium contrast necessary in all cases to delineate the nature of an IF, it is likely that further studies in the ESRD population may require follow-up imaging with computed tomography with iodinated contrast to follow up on indeterminate findings of non-contrast CMR.

\section{Limitations}

Our study is a retrospective study using historical images. Given the number and significance of findings discovered, a prospective study would be ethically entirely inappropriate.

Undoubtedly methods of determining what constitutes an IF will vary slightly between studies and reporters, and it has not been possible to adjust for this.

In studies such as ours, where a further focused reading of the CMR images has taken place, it might be expected that the rate of findings would be slightly greater than in other studies. For example, Wyttenbach [27] found the rate of significant IF identified increased from $11.7 \%$ to $20.5 \%$ when the same clinician re-interpreted historical clinical scans specifically looking for extra-cardiac IF. However, this does not detract from the overall value of this study, which highlights that IF in research CMR in the ESRD population are both common and important.

\section{Conclusions}

This study of 161 CMR images performed for research purposes in the CKD5 population revealed 150 IF. $14.9 \%$ of the participants had a new significant IF that warranted further investigation or a change in their management.

The prevalence of IF in the ESRD population is such that all investigators planning studies undertaking CMR must take proper account of it. All investigators should consult with experienced radiologists early in the trial planning phase to ensure an appropriate mechanism for reporting is in place. Funding for radiologist reporting should be factored into grant applications. All images obtained should be reported by a qualified radiologist within a clinically acceptable timeframe.

All potential participants in renal CMR research studies should be counselled about the frequency and implications of possible IF and protocols for managing IF should be part of good clinical research practice.

Acknowledgements The scientific guarantor of this publication is Dr Patrick Mark. The authors of this manuscript declare no relationships with any companies, whose products or services may be related to the subject matter of the article. The original research was funded by the British Heart Foundation. JRWM is supported by the Wellcome Trust through the Scottish Translational Medicine and 
Therapeutics initiative (Grant. WT 085664) in the form of a Clinical Research Fellowship. No complex statistical methods were necessary for this paper. Institutional Review Board approval was obtained for the original study and was not required for this retrospective analysis. Written informed consent was obtained from all subjects (patients) in the original study. Methodology: retrospective, observational, single centre study.

Open Access This article is distributed under the terms of the Creative Commons Attribution 4.0 International License (http:// creativecommons.org/licenses/by/4.0/), which permits unrestricted use, distribution, and reproduction in any medium, provided you give appropriate credit to the original author(s) and the source, provide a link to the Creative Commons license, and indicate if changes were made.

\section{References}

1. Levy D, Garrison RJ, Savage DD, Kannel WB, Castelli WP (1990) Prognostic implications of echocardiographically determined left ventricular mass in the Framingham Heart Study. N Engl J Med 322:1561-1566

2. Levin A, Djurdjev O, Thompson C, Barrett B, Ethier J, Carlisle E et al (2005) Canadian randomized trial of hemoglobin maintenance to prevent or delay left ventricular mass growth in patients with CKD. Am J Kidney Dis 46:799-811

3. Thadhani R, Appelbaum E, Pritchett Y, Chang Y, Wenger J, Tamez $\mathrm{H}$ et al (2012) Vitamin D therapy and cardiac structure and function in patients with chronic kidney disease: the PRIMO randomized controlled trial. JAMA 307:674-684

4. Wang AY-M, Fang F, Chan J, Wen Y-Y, Qing S, Chan IH-S et al (2014) Effect of paricalcitol on left ventricular mass and function in CKD-the OPERA trial. J Am Soc Nephrol 25:175-186

5. Culleton B, Walsh M, Quinn R, Donnelly S, Friedrich M, Kumar A (2007) Effect of frequent nocturnal hemodialysis vs conventional hemodialysis. J Am Med Assoc 298:1291-1299

6. In-center hemodialysis six times per week versus three times per week — NEJM n.d. doi:10.1056/NEJMoa1001593. Accessed 18 Aug 2015

7. Chan CT, Greene T, Chertow GM, Kliger AS, Stokes JB, Beck GJ et al (2013) Effects of frequent hemodialysis on ventricular volumes and left ventricular remodeling. Clin J Am Soc Nephrol 8:2106-2116

8. Patel RK, Mark PB, Halliday C, Steedman T, Dargie HJ, Cobbe SM et al (2011) Microvolt T-wave alternans in end-stage renal disease patients-associations with uremic cardiomyopathy. Clin J Am Soc Nephrol 6:519-527

9. Mark PB, Johnston N, Groenning BA, Foster JE, Blyth KG, Martin TN et al (2006) Redefinition of uremic cardiomyopathy by contrastenhanced cardiac magnetic resonance imaging. Kidney Int 69: 1839-1845

10. Chan PG, Smith MP, Hauser TH, Yeon SB, Appelbaum E, Rofsky $\mathrm{NM}$ et al (2009) Noncardiac pathology on clinical cardiac magnetic resonance imaging. JACC Cardiovasc Imaging 2:980-986

11. Irwin RB, Newton T, Peebles C, Borg A, Clark D, Miller C et al (2013) Incidental extra-cardiac findings on clinical CMR. Eur Heart J Cardiovasc Imaging 14:158-166
12. Atalay MK, Prince EA, Pearson CA, Chang KJ (2011) The prevalence and clinical significance of noncardiac findings on cardiac MRI. Am J Roentgenol 196:387-393

13. Sohns JM, Schwarz A, Menke J, Staab W, Spiro JE, Lotz J et al (2014) Prevalence and clinical relevance of extracardiac findings at cardiac MRI. J Magn Reson Imaging 39:68-76

14. McKenna DA, Laxpati M, Colletti PM (2008) The prevalence of incidental findings at cardiac MRI. Open Cardiovasc Med J 2:20-25

15. Management of incidental findings detected during research imaging | The Royal College of radiologists n.d. https://www.rcr.ac.uk/ management-incidental-findings-detected-during-researchimaging. Accessed 29 June 2015

16. Patel RK, Mark PB, Johnston N, McGregor E, Dargie HJ, Jardine AG (2008) Renal transplantation is not associated with regression of left ventricular hypertrophy: a magnetic resonance study. Clin J Am Soc Nephrol 3:1807-1811

17. Stewart GA, Mark PB, Johnston N, Foster JE, Cowan M, Rodger RSC et al (2004) Determinants of hypertension and left ventricular function in end stage renal failure: a pilot study using cardiovascular magnetic resonance imaging. Clin Physiol Funct Imaging 24:387-393

18. Swaminathan S, Shah SV (2007) New insights into nephrogenic systemic fibrosis. J Am Soc Nephrol 18:2636-2643

19. Grobner T (2006) Gadolinium-a specific trigger for the development of nephrogenic fibrosing dermopathy and nephrogenic systemic fibrosis? Nephrol Dial Transplant 21:1104-1108

20. Edwards NC, Moody WE, Chue CD, Ferro CJ, Townend JN, Steeds RP (2014) Defining the natural history of uremic cardiomyopathy in chronic kidney disease: the role of cardiovascular magnetic resonance. JACC Cardiovasc Imaging 7:703-714

21. Loy A, Morgan R, O’Dea S, Daly C, Mulcahy F (2015) Clinically significant extra-cardiac findings in asymptomatic HIV-positive men undergoing cardiac magnetic resonance imaging. Int J STD AIDS 26:346-351

22. (2005) K/DOQI clinical practice guidelines for cardiovascular disease in dialysis patients. Am J Kidney Dis 45:16-153

23. London GM, Pannier B, Guerin AP, Blacher J, Marchais SJ, Darne B et al (2001) Alterations of left ventricular hypertrophy in and survival of patients receiving hemodialysis: follow-up of an interventional study. J Am Soc Nephrol 12:2759-2767

24. Jakubovic BD, Wald R, Goldstein MB, Leong-Poi H, Yuen DA, Perl J et al (2013) Comparative assessment of 2-dimensional echocardiography vs cardiac magnetic resonance imaging in measuring left ventricular mass in patients with and without end-stage renal disease. Can J Cardiol 29:384-390

25. Stewart GA, Foster J, Cowan M, Rooney E, McDonagh T, Dargie HJ et al (1999) Echocardiography overestimates left ventricular mass in hemodialysis patients relative to magnetic resonance imaging. Kidney Int 56:2248-2253

26. Marckmann P, Skov L, Rossen K, Dupont A, Damholt MB, Heaf JG et al (2006) Nephrogenic systemic fibrosis: suspected causative role of gadodiamide used for contrast-enhanced magnetic resonance imaging. J Am Soc Nephrol 17:23592362

27. Wyttenbach R, Médioni N, Santini P, Vock P, Szucs-Farkas Z (2012) Extracardiac findings detected by cardiac magnetic resonance imaging. Eur Radiol 22:1295-1302 